\title{
Intelligent Fault Identification System for Transmission Lines Using Artificial Neural Network
}

\author{
Seema Singh ${ }^{1}$, Mamatha $\mathrm{K} \mathrm{R}^{2}$, Thejaswini $\mathrm{S}^{3}$ \\ ${ }^{I}$ (Associate Professor, Dept. of Electronics \& Comm. Engg., BMS Institute of Technology, Bangalore, India) \\ ${ }^{2}$ (Assistant Professor, Dept. of Electronics \& Comm. Engg., BMS Institute of Technology, Bangalore, India) \\ ${ }^{3}$ (Assistant Professor, Dept. of Telecommunication Engg., BMS Institute of Technology, Bangalore, India)
}

\begin{abstract}
Transmission and distribution lines are vital links between generating units and consumers. They are exposed to atmosphere, hence chances of occurrence of fault in transmission line is very high, which has to be immediately taken care of in order to minimize damage caused by it. This paper focuses on detecting the faults on electric power transmission lines using artificial neural networks. A feed forward neural network is employed, which is trained with back propagation algorithm. Analysis on neural networks with varying number of hidden layers and neurons per hidden layer has been provided to validate the choice of the neural networks in each step. The developed neural network is capable of detecting single line to ground and double line to ground for all the three phases. Simulation is done using MATLAB Simulink to demonstrate that artificial neural network based method are efficient in detecting faults on transmission lines and achieve satisfactory performances. A $300 \mathrm{~km}, 25 \mathrm{kv}$ transmission line is used to validate the proposed fault detection system. Hardware implementation of neural network is done on TMS320C6713.
\end{abstract}

Keywords: Transmission Line, Asymmetric fault detection, Artificial neural network (ANN), Back Propagation algorithm, DSP processor TMS320C6713, Code Composer Studio, MATLAB/Simulink

\section{Introduction}

The advent of large generating stations and highly interconnected transmission lines makes early fault detection and rapid equipment isolation imperative to maintain system stability. Transmission line is used to transfer power or voltage to long distance destination. Power or voltage generated from source is supplied to the load through the Transmission Line. While transmitting, Transmission Line encounters various faults due to momentary tree contact, a bird or an animal contact or due to other natural reasons such as thunderstorms or lightning.

Earlier systems use conventional method for the fault detection which results in the late detection and inaccurate results [1]-[5]. Conventional algorithms are based on deterministic computations on a well-defined model for transmission line protection. Bergon model was extensively used to model transmission line. Conventional distance relays consider power swing as a fault and tripping because of such malfunctioning would lead to serious consequences for power system stability. To improve the performance, Neural Network architecture is used which results in the earlier fault detection.

From quite a few years, intelligent based methods are being used in the process of fault detection. Three major artificial intelligence based techniques that have been widely used in the power and automation industry are:

- Expert System Techniques

- Artificial Neural Networks

- Fuzzy Logic Systems

Ref [6] used an adaptive neuro-fuzzy approach to develop an inference system for transmission line fault classification and location. It incorporates the effects of power swings. Fault location is done using wavelet-neuro-fuzzy combined approach. The wavelet transform captures the dynamic characteristics of fault signals using wavelet multi-resolution analysis (MRA) coefficients. The fuzzy inference system (FIS) and the adaptive-neuro-fuzzy inference system (ANFIS) are both used to extract important features from wavelet MRA coefficients and thereby to reach conclusions regarding fault location. However, combination of different approaches makes the system complicated. This work uses ANN based approach which uses the fluctuations in current level as the key feature to detect faults. Neural network is capable of working with real time data and responses to the changes in surrounding environment immediately and this makes the system more flexible for the fault detection.

ANN based methods do not require a knowledge base for the detection of faults unlike the other artificial intelligence based methods. The prime motive behind this work is that a very accurate fault detector could make if employed in a power transmission and distribution system, in terms of the amount of money and 
time that can be saved. The main goal of Fault Detection is to detect a fault in the power system with the highest practically achievable accuracy. When the physical dimensions and the size of the transmission lines are considered, the accuracy with which the designed fault detector detects faults in the power system becomes very important.

Special features of ANN for fault tolerant systems:

- ANN is made of massive interconnection of elementary processing units, information processing can be carried out in a parallel distributed manner. This makes real time processing of large volumes of data more readily realizable.

- ANN can model any degree of nonlinearity and thus are useful in solving these problems which are inherently nonlinear.

- ANN approach is non-algorithmic and requires no prior knowledge functions relating the problem variables. Also being non algorithmic, they do not make any approximations unlike as the case with most of the mathematical models.

- ANN is capable of handling situations of incomplete information, corrupt data and thus is highly fault tolerant.

As power system grow both in size and complexity, it becomes necessary to identify different faults faster and more accurately using more powerful algorithms. Back propagation algorithm of neural network is used for the fault diagnosis system. Different fault like single line to ground fault and double line to ground faults are detected using ANN. Fault detector module of a transmission line protective scheme can be used to start other relaying modules.

The basic block diagram of the work is shown in Figure 1. The Simulink model of $300 \mathrm{~km}$ transmission line with sampling time $0.02 \mathrm{~s}$ is used for simulation.

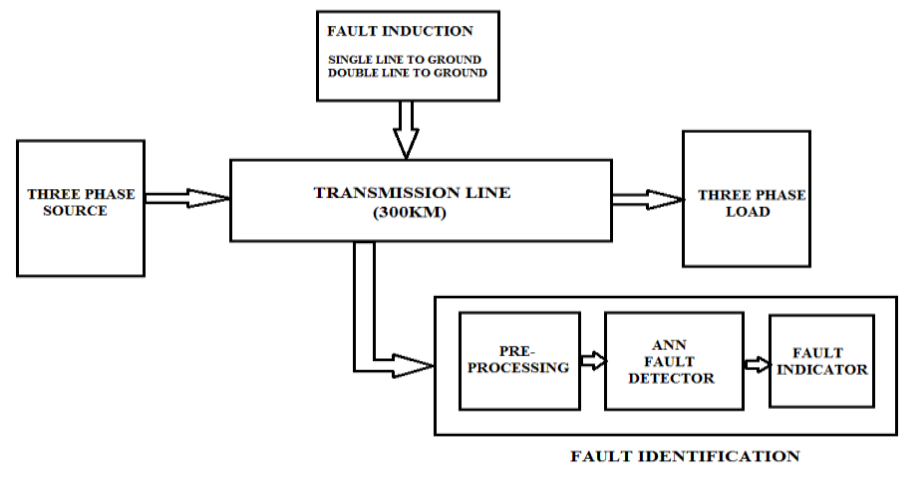

Fig 1: Block diagram for fault identification in transmission line using ANN

Simulink model of transmission line is used for validation of neural network based fault detection system. Neural networks have an inherent parallel architecture with multiple input and output nodes, it can be implemented to high speed parallel hardware with multivariable input and output. Neural network model in this paper is implemented using DSP processor TMS320C6713.

\section{Transmission line and asymmetric fault}

Transmission lines are used for long distance power supply. Transmission lines, when interconnected with each other, become transmission networks. High-voltage overhead conductors are not covered by insulation. The conductor material is nearly always an aluminum alloy, made into several strands and possibly reinforced with steel strands. Copper was sometimes used for overhead transmission but aluminum is lighter, yields only marginally reduced performance, and costs much less. Overhead conductors are a commodity supplied by several companies worldwide. Improved conductor material and shapes are regularly used to allow increased capacity and modernize transmission circuits. High-voltage direct current (HVDC) is used to transmit large amounts of power over long distances or for interconnections between asynchronous grids. The Transmission line model, three phase source and three phase load is simulated using MATLAB/Simulink powergui. Sampling is done to reduce complexity and increase simulation speed.

In an electric power system, a fault is any abnormal electric current. For example, a short circuit is a fault in which current bypasses the normal load. An open-circuit fault occurs if a circuit is interrupted by some failure. In three-phase systems, a fault may involve one or more phases and ground, or may occur only between 
phases. In a "ground fault" or "earth fault", charge flows into the earth. The prospective short circuit current of a fault can be calculated for power systems. In power systems, protective devices detect fault conditions and operate circuit breakers and other devices to limit the loss of service due to a failure.

An asymmetric or unbalanced fault does not affect each of the three phases equally. There are three common types of asymmetric faults, namely line to line, line to ground and double line to ground. Line-to-line fault is a short circuit between lines, caused by ionization of air, or when lines come into physical contact, for example due to a broken insulator. Line-to-ground - is a short circuit between one line and ground, very often caused by physical contact, for example due to lightning or other storm damage. Double line-to-ground fault is where two lines come into contact with the ground (and each other), also commonly due to storm damage. Single line to ground and double line to ground fault is simulated using three phase fault block. It is capable of introducing short circuit fault at the different interval of time. It can be visualized using the waveform of the input and output current and voltages through scope.

\section{ANN based fault detection system}

Artificial intelligence, cognitive modeling, and neural networks are information processing paradigms inspired by the way biological neural systems process data. Artificial intelligence and cognitive modeling try to simulate some properties of biological neural networks. Artificial neural networks have been applied successfully to speech recognition, image analysis and adaptive control, in order to construct software agents (in computer and video games) or autonomous robots and specially in fault detection system [16], [17]. Neural network theory has served both to better identify how the neurons in the brain function and to provide the basis for efforts to create artificial intelligence. Fig 2 shows a single neuron. The following diagram shows a simple neuron with:

Neuron consists of three basic components, namely weights, thresholds/biases and a single activation function. Values $\mathrm{w}_{1}, \mathrm{w}_{2} \ldots \ldots \mathrm{w}_{\mathrm{n}}$ are weights to determine the strength of input vector $\mathrm{X}=\left[\mathrm{x}_{1}, \mathrm{x}_{2}, \ldots \ldots, \mathrm{x}_{\mathrm{n}}\right]^{\mathrm{T}}$. Each input is multiplied with its associated weight of the neuron XT.W.

$$
\mathrm{I}=\mathrm{X}^{\mathrm{T}} \cdot \mathrm{W}=\mathrm{x}_{1} \mathrm{~W}_{1}+\mathrm{x}_{2} \mathrm{w}_{2}+\ldots \ldots+\mathrm{x}_{\mathrm{n}} \mathrm{W}_{\mathrm{n}}=\sum_{i=1}^{n} x_{i} w_{i}
$$

Threshold, $\varphi$ is the neuron's internal offset. The neuron fire or produces positive output only if the total input $\mathrm{I}$ is above the threshold value. It affects the activation of the node output $\mathrm{y}$ as

$$
\mathrm{Y}=\mathrm{f}(\mathrm{I})=\mathrm{f}\left\{\sum_{i=1}^{n} x_{i} w_{i}-\varphi_{k}\right\}
$$

To generate the final output $\mathrm{Y}$, the sum is passed on to a non- linear filter $\mathrm{f}$ called activation function or transfer function, which releases the output Y. There are various activation functions which are chosen depending on the type of problem to be solved by the network. The most common activation function includes linear function, tangent hyperbolic function, threshold function and sigmoidal function. Most popular sigmoidal function follows the transition equation shown below.

$$
\mathrm{Y}=\mathrm{f}(\mathrm{I})=\frac{1}{1+e^{-\alpha l}} \text {, where } \alpha \text { is the slope of sigmoidal function followed. }
$$
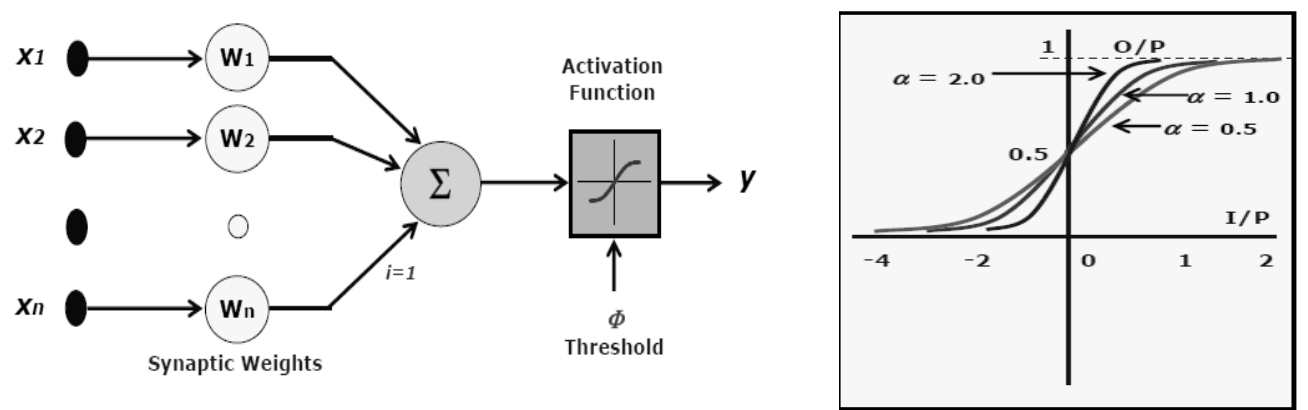

Fig. 2: A single neuron and sigmoidal activation function

The capability of the neural network increases as number of neurons increases. This capability multiplies as number of layers in neural network structure increases. Figure 3 shows multilayer neural network with one hidden layer. The weights connecting neurons are varied continuously while training the neural network. In NN applications, the challenge is to find the right values for the weights and the threshold. Various algorithms are developed in neural network field depending on different problems and applications where it has been used. Back Propagation, Radial Basis Functions, Multi-Layer Perceptron algorithm, Adaptive Resonance Theory (ART), Self Organizing Maps (SOM) and Counter Propagation Networks (CPN) are few algorithms of neural networks.

Various neural network architecture, training algorithms and transfer functions were studied to decide upon the final neural network model for fault detection system. Back propagation algorithm, a supervised learning is used as the network will be trained using the data created from the simulation model of transmission. 
Number of hidden layers and number of neurons in each layer can be varied depending on the complexity of the problem to be solved.

The training data was created using Simulink model of $300 \mathrm{~km}$ long transmission line for various fault cases. This training data consists of various vectors of the output and input current and voltage. Using these values and the created target vector, neural network is trained to detect the fault at the different instants of time. Training is done using back propagation training algorithm using "traingd" function of neural network toolbox of MATLAB. The trained/updated neural network model for fault detection is converted to Simulink block using neural network toolbox. This is done to make it usable with Simulink model of transmission line.

\subsection{Training of the Neural Network}

The neural network architecture with three hidden layers was fixed for the simulation purpose. Hidden layers consist of 7-10-3 neurons as shown in Fig. 3. Input layer consists of three neurons which takes the current of three phases of the transmission line. Output layer consists of one neuron which indicates if fault is there. A threshold of 0.9 is fixed based on simulation in order to avoid false alarms. As shown in figure, the training algorithm used is gradient descent variant of back propagation algorithm. Figure shows the number of iteration, time taken to train the Neural Network. It also shows the different performance plots of the Neural Network after training of the network.

The different training parameter encountered during the training process is gradient, mean square error and validation check. For better training purpose, mean square error value should decrease when the training process continues; validation check gives us maximum number of fails in the Neural Network training process. These training parameter plots are shown in Fig. 4.

\subsection{Performance Plot}

As discussed earlier in this section, the training data is created using simulation model of transmission lines. Single line to ground and double line to ground fault was introduced in different phases at different time instants. This training data, when fed for training the neural network, is divided into three parts. The data samples are divided into train, test and validation sets randomly by the program, in the ratio 0.7:0.15:0.15.

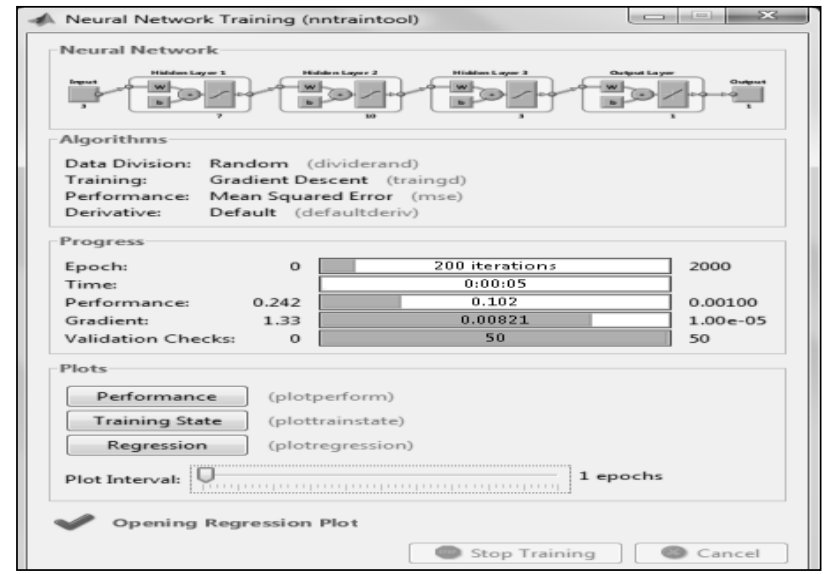

Fig 3: Neural network training in Simulink tool
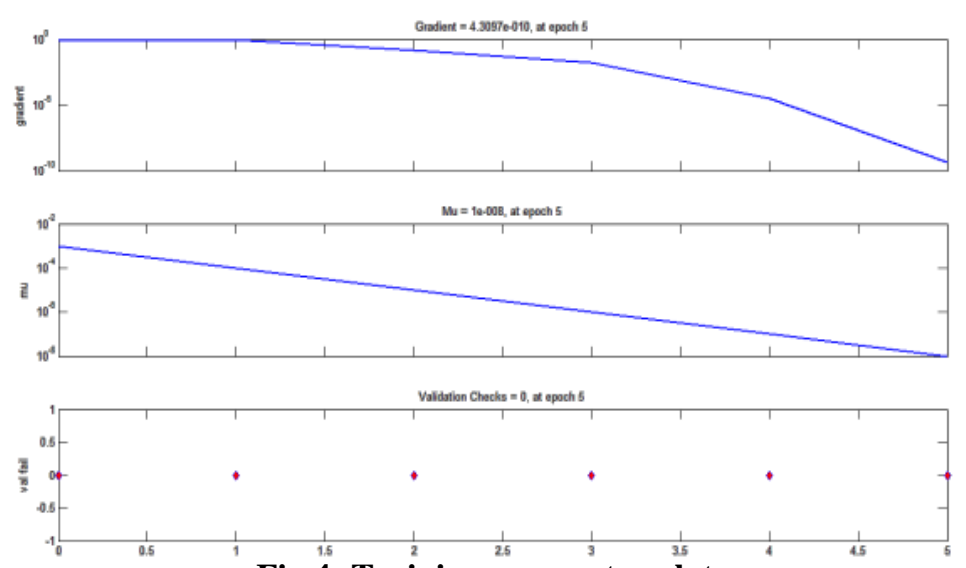

Fig 4: Training parameter plots 
The figure 5 shows the performance of Neural Network when Neural Network is trained with particular input vectors and target vectors. The plot shows that the variation of different parameters throughout the training process.

After training is completed satisfactorily, the neural network is tested with the available sample data to evaluate the performance of the updated trained neural network. If the performance is not up to the expectations, some variations may me experimented with the neural network structure by varying number of hidden layers of number of neurons in each layer. Different training algorithm may also be experimented with various activation functions.

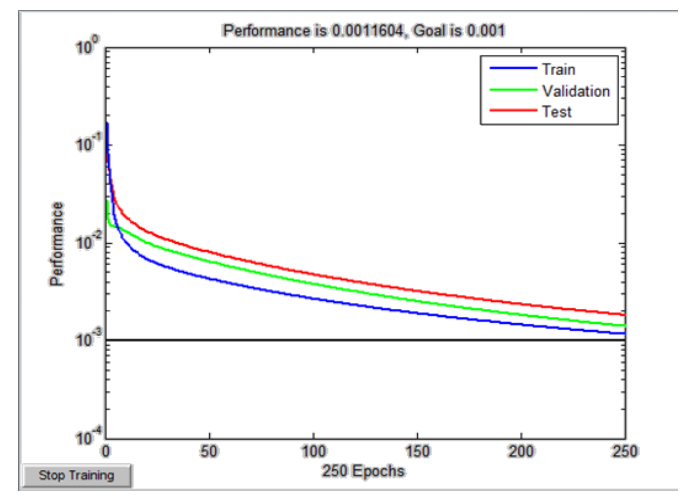

Fig 5: Performance plot

Once the neural network with satisfactory performance is developed, its Simulink model is developed using 'gensim' function so that it can be used with other block in the simulation model. Neural network simulation model is shown in Fig. 6. The complete simulation set up is shown in Fig. 7.

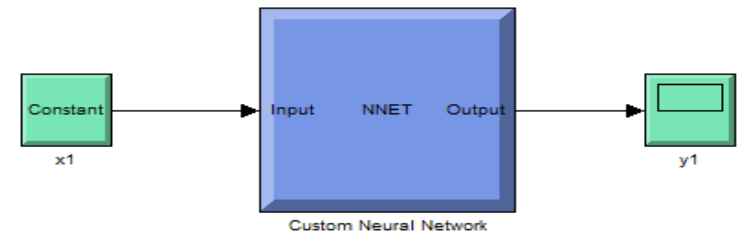

Fig 6: Simulink model of developed neural network

\section{Simulation Set-up and Hardware Implementation}

As discussed in previous section, transmission line with three phase source and load, three phase fault and neural network based fault detection simulation models are developed to test the capability of the proposed method. Transmission line model is generated with discrete state and pi section line. Using this model samples are reduced from 1000 samples to 50 samples, which is feasible for real time application and easy for implementation. Sampling time of this model is $\mathrm{Ts}=0.02 \mathrm{~ms}$ and simulation is done for 1 sec. With these specifications, the current and voltage vectors consist of 51 samples. This simulation model of transmission line with various fault cases is used to create training data which was used to train neural network.

A feed forward neural network with 3 hidden layers is used for fault detection purpose. The number of neurons in all hidden layer can be varied to get the optimum performance. First hidden layer is fixed with 7 neurons, second with 10 neurons and last hidden layer with 3 neurons as shown in Fig. 3. Output layer has one neuron, output indicates whether fault has occurred or not at different instants of time. This neural network structure is trained using back propagation training algorithm for the training data of transmission line model. This trained/updated neural network is capable of detecting faults. Detection of fault is successfully done for single line to ground and double line to line ground for all the three phases.

The fault detection system successfully detects single line and double line to ground fault in transmission line.

\section{Hardware Implementation:}

Fault detection system using neural network is implemented on DSP processor. Neural network model parameters are studied and programmed using ' $\mathrm{C}$ ' language and the same is implemented in TMS320C6713 using code composer studio. DSP processor is used to verify the result for timing constraints.

Implementation of Neural Network on DSP Processor TMS320C6713 is difficult, because of the size of the generated Neural Network. To overcome the difficulties faced while doing implementation, Neural Network was modified as mentioned below: 
- Changing the input from floating point to the binary form. For eg. Input in floating point $=0.04$ is changed to the input in binary form $=0$ and Input in floating point $=0.92$ is changed to the input in binary form $=1$.As detection of line to ground fault is short circuit fault, input can be transformed to binary values.

- The size of the neural network is reduced, by changing the number of hidden layer to 1 and number of neurons in the hidden layer to 4 . This neural network could give satisfactory results for the transformed binary values.

- Implementation of this neural network with one hidden layer of 4 neurons is preferred for the hardware, which contains 16 weight values and 5 biases. Totally 21 values are required, which is easier for hardware implementation and able to detect faults successfully.

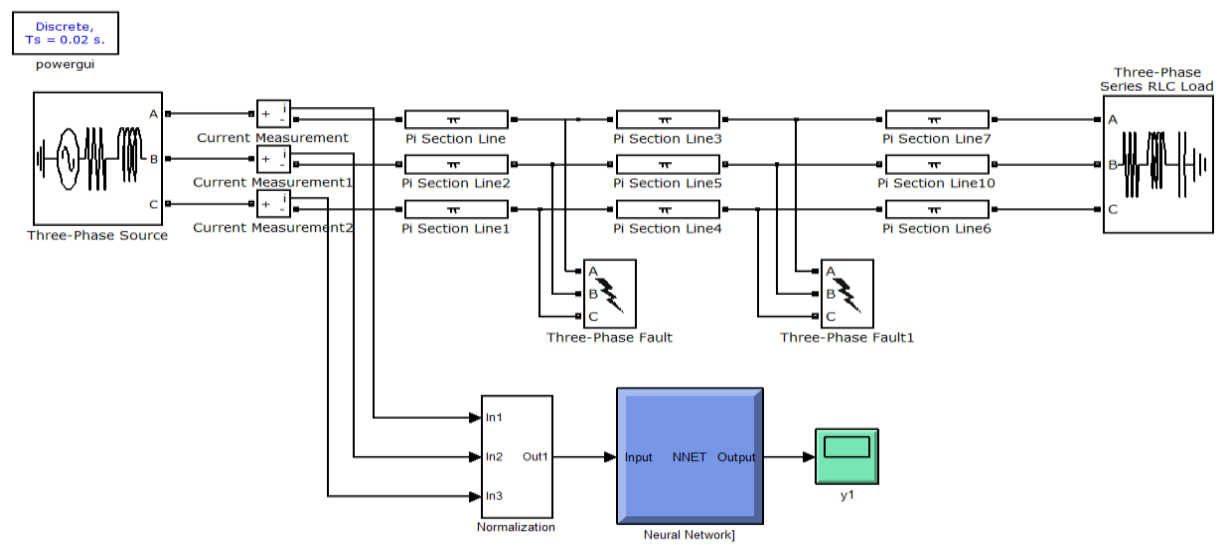

Fig 7: Simulink model of Intelligent Fault identification system in transmission line model

\section{Discussion Of The Results}

In this work, an attempt was made to develop an intelligent fault identification system for transmission lines using artificial neural network. Three phase current and voltages are in analog form and the waveform in its analog form is shown in Fig. 8. It shows the continuous signals for all the three phases of the voltage before sampling.

However, to design a fast and accurate fault detection system, it is important to sample it. The sampled waveform at $0.2 \mathrm{~s}$ is shown in Fig. 9 for $1 \mathrm{~s}$. This figure shows the discrete signals for all the three phases of the voltages after sampling.

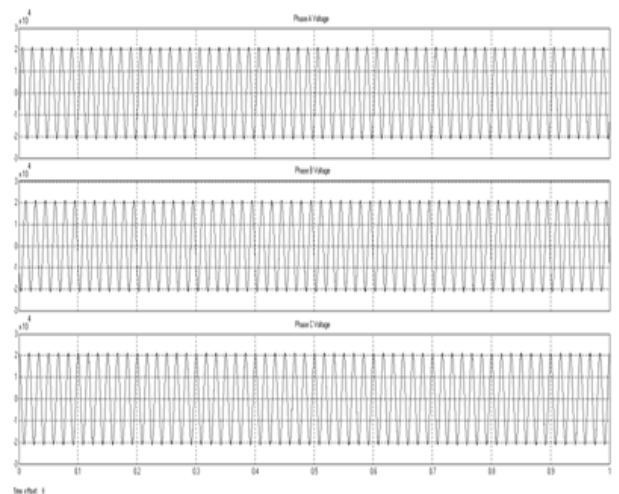

Fig 8: Waveform before sampling

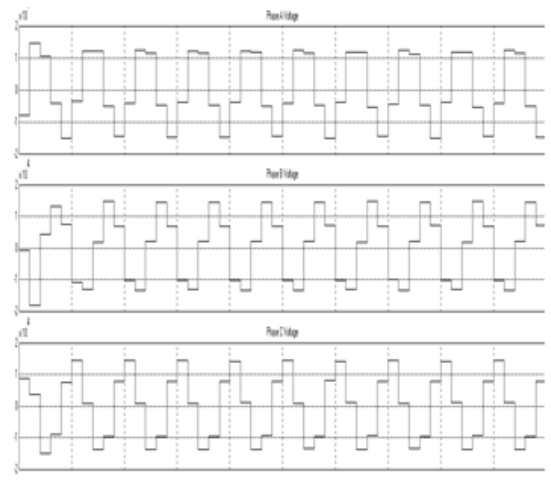

Fig 9 : Waveform after sampling

Various cases of single line to ground and double line to ground fault is tested for the developed fault detection system. Test cases are listed in the table 1 and corresponding plots are shown in Fig. 10 to 21 . Each figure has four sub plots. First three sub plots show current levels at phase A, B and C. Fourth plot show neural network output, which indicates the occurrence of fault by switching to a high level. Fault in one phase disturbs other phase current as shown in all the result plots. Fig.22 shows fault detection from neural network implemented in DSP Processor TMS320C6713.A slight delay is seen in the output when it is switching to ' 1 ' to show occurrence of fault. This happens due to hardware delay. 


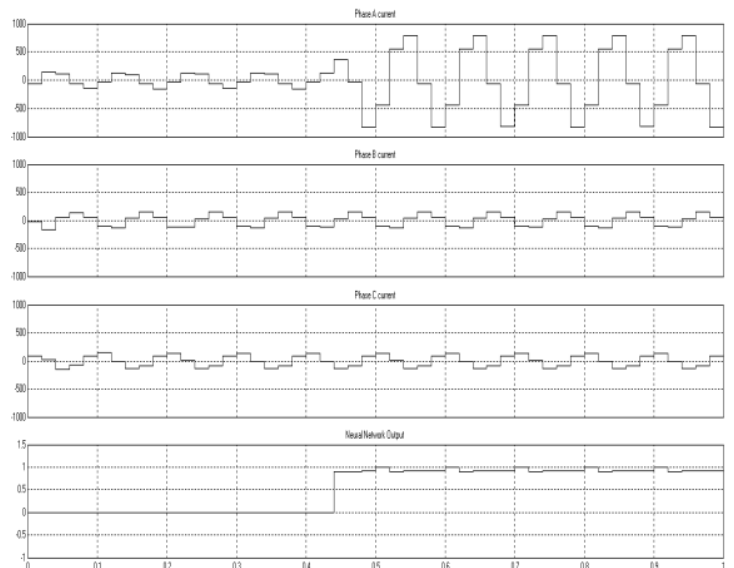

Fig 10: Fault detection for Single line to ground fault in phase $A$ at $4 \mathrm{~s}$

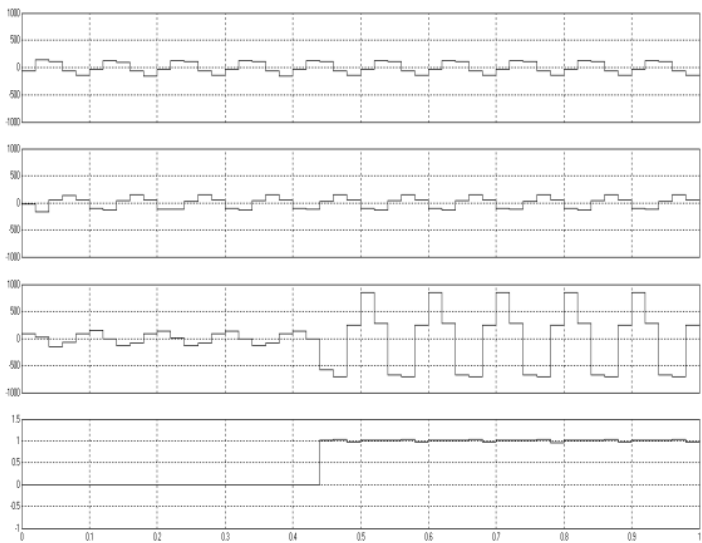

Fig 12: Fault detection for Single line to ground fault in phase $C$ at $4 \mathrm{~s}$

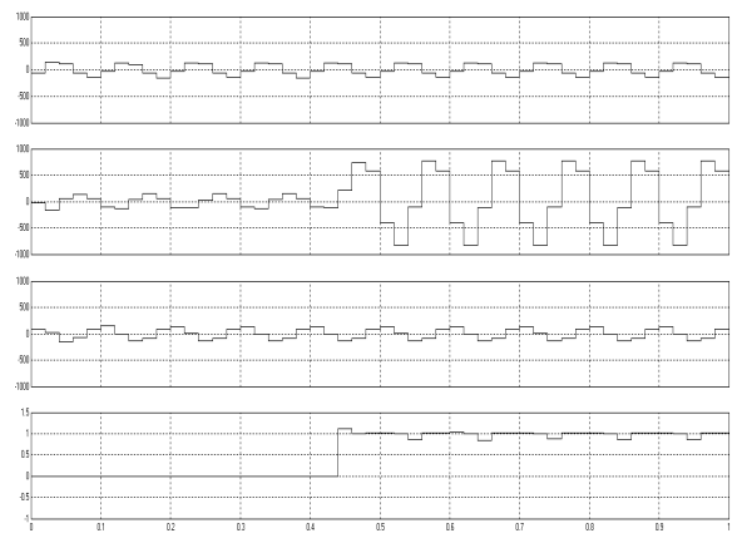

Fig 11: Fault detection for Single line to ground fault in phase $B$ at $4 s$

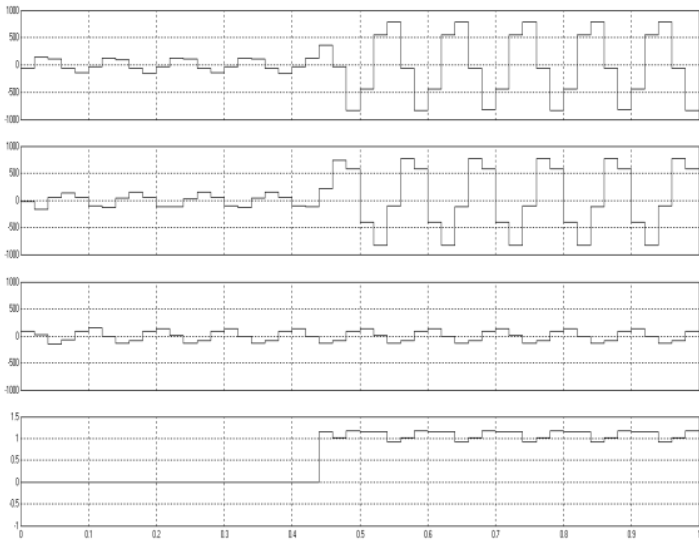

Fig 13: Fault detection for double line to ground (Phase A and B) Fault at 4s

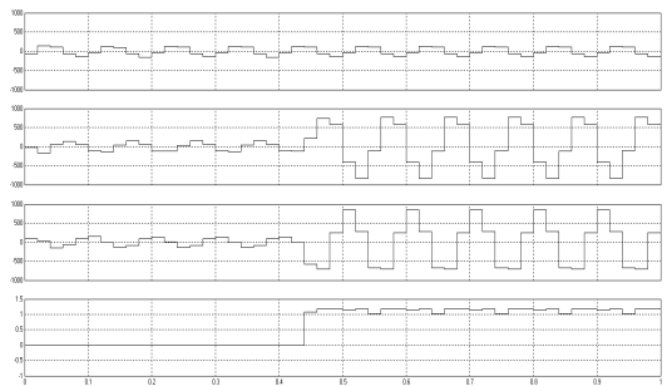

Fig 14: Fault detection for double line to ground (Phase B and C) Fault at 4s

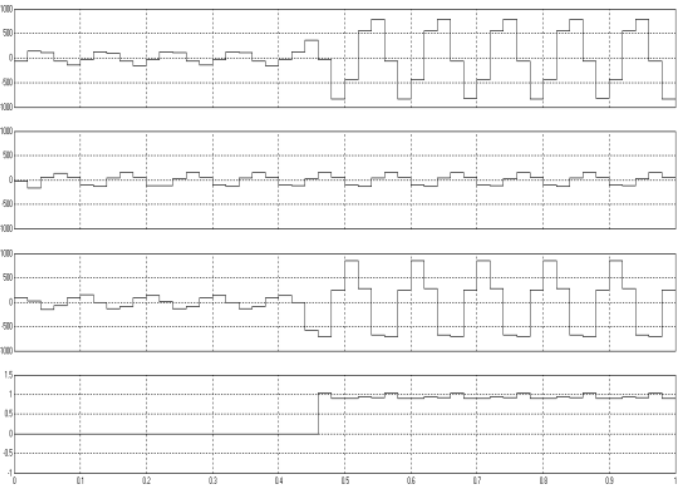

Fig 15: Fault detection for double line to ground (Phase A and C) Fault at 4s

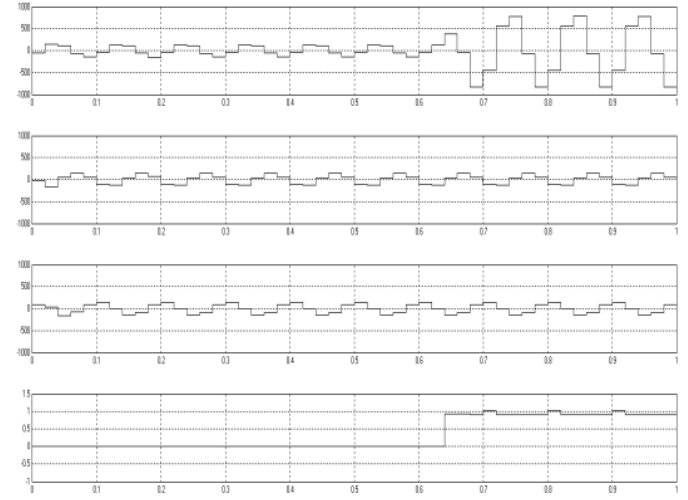

Fig 16: Fault detection for Single line to ground Fault in Phase A at $6 \mathrm{~s}$ 


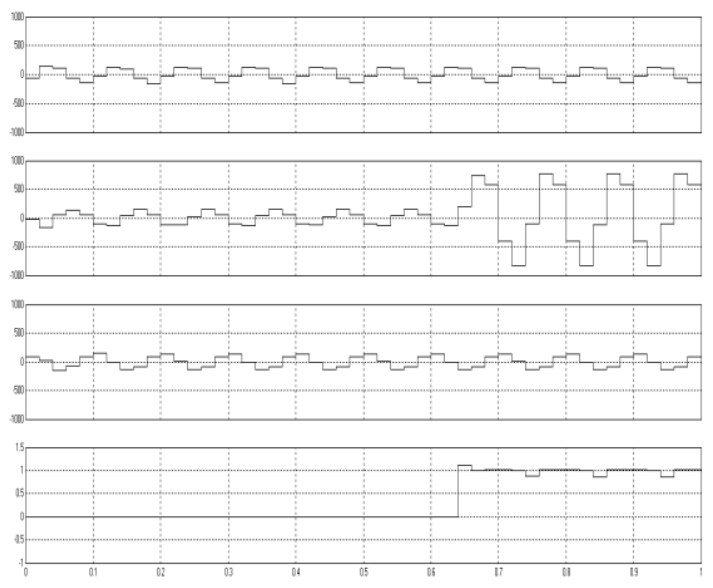

Fig 17: Fault detection for Single line to ground Fault in Phase $B$ at $6 \mathrm{~s}$

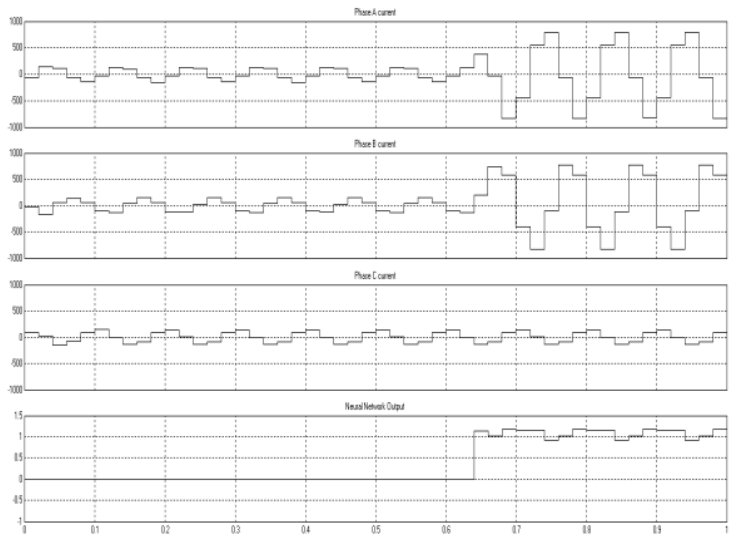

Fig 19: Fault detection for double line to ground (Phase A and B) Fault at 6s

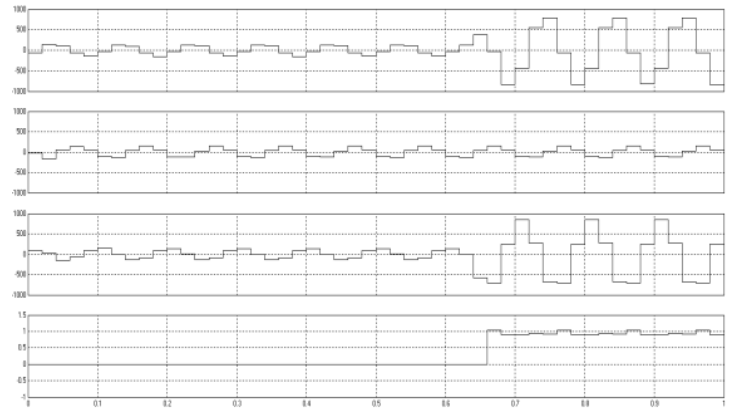

Fig 21: Fault detection for double line to ground (Phase A and C) Fault at 6s

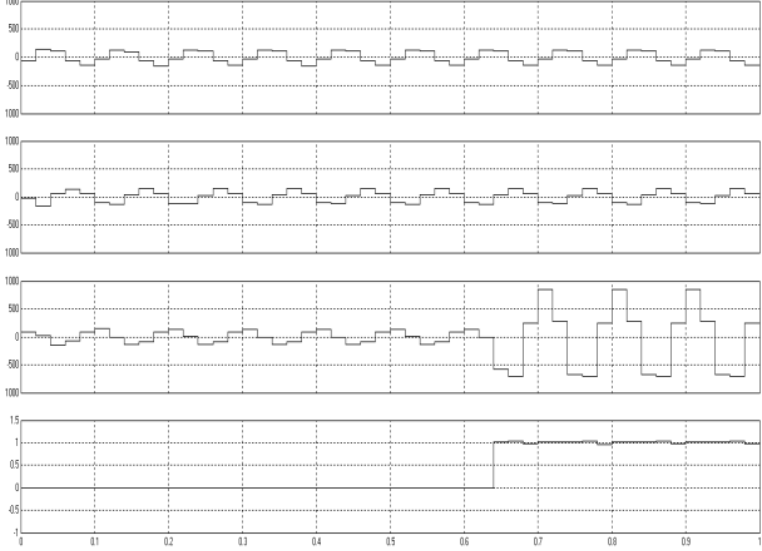

Fig 18: Fault detection for Single line to ground Fault in Phase $\mathrm{C}$ at $6 \mathrm{~s}$

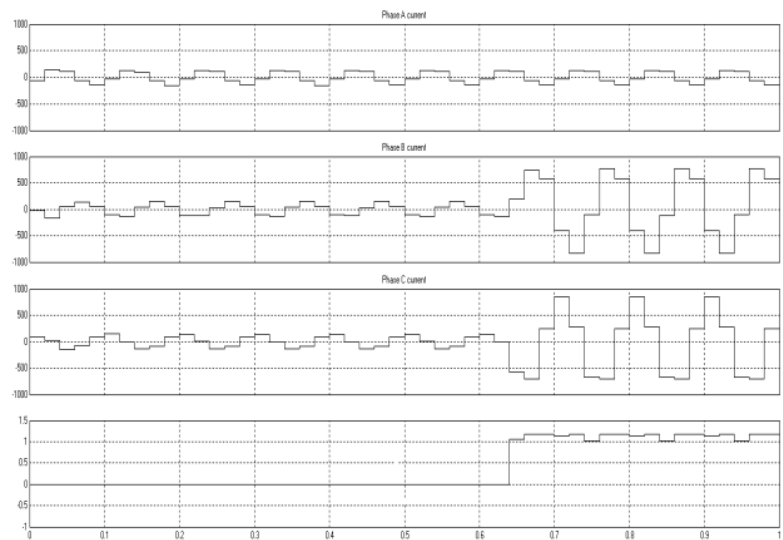

Fig 20: Fault detection for double line to ground (Phase B and C) Fault at 6s

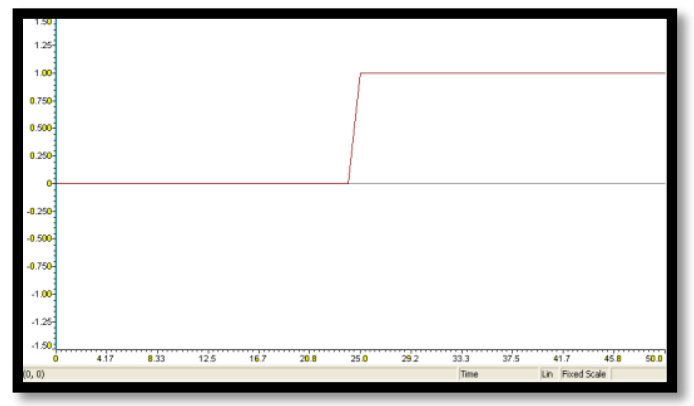

Fig 22: Neural network output from DSP Processor

Neural network is generalized and thus capable of finding any type of fault in the transmission line. The trained Neural Network can detect Single line to ground and Double line to ground fault. Effects of the different system parameters and conditions are studied. Extensive studies indicate that the network is able to detect faults earlier and correctly and its performance is not affected by the changing network conditions.

\section{CONCLUSION}

The proposed method uses an artificial neural network-based scheme for fast and reliable fault detection. Various Asymmetric fault (Single line to ground and double line to ground fault) are simulated and an ANN based algorithm is used for detection of these faults. Performance of the proposed scheme is evaluated using various fault types and encouraging results are obtained. 
There are many applications for which the Digital Signal Processor becomes an ideal choice as they provide the best possible combination of performance, power and cost. Most of the DSP applications can be simplified into multiplications and additions, so the MAC (Multiply- Accumulate) formed a main functional unit in early DSP processors. Neural network is a mathematical structure with combination of parallel MAC operation in its each layer with different transfer functions like sigmoid and linear functions. Thus neural network can be efficiently implemented on DSP processor using CC Studio as done in this work. Thus neural network based analysis provides the solution much faster than the conventional methods without degrading the accuracy.

Further work can be carried out by developing detection system to detect other asymmetric and symmetric fault. Hardware implementation can be extended to more complex neural networks with higher version of DSPs. FPGA may also be another alternative for hardware implementation with a comparative analysis of the two possibilities of hardware.

\section{Acknowledgements}

The authors thank the students, Mamta Devi, Mohanakalyani B N, Pratibha C and Nagashree K Rao, who played an important role in carrying out this work. The authors are also thankful to KSCST, Bangalore for selecting and sponsoring this work.

\section{REFERENCES}

[1] Das R, Novosel D, "Review of fault location techniques for transmission and sub - transmission lines", proceedings of 54th Annual Georgia Tech Protective RelayingConference, 2000.

[2] IEEE guide for determining fault location on AC transmission and distribution lines.IEEE Power Engineering Society Publ., New York, IEEE Std C37.114, 2005.

[3] Saha MM, Izykowski J, Rosolowski E, "Fault Location on Power Networks", Springer publications, 2010.

[4] Magnago FH, Abur A, "Advanced techniques for transmission and distribution system fault location", Proceedings of CIGRE Study committee 34 Colloquium and Meeting, Florence, 1999, paper 215.

[5] Tang Y, Wang HF, Aggarwal R K, "Fault indicators in transmission and distribution systems", Proceedings of International conference on Electric Utility Deregulation and Restructuring and Power Technologies - DRPT, 2000, pp. 238-243.

[6] Reddy MJ, Mohanta DK, “Adaptive-neuro-fuzzy inference system approach for transmission line fault classification and location incorporating effects of power swings", Proceedings of IET Generation, Transmission and Distribution, 2008, pp. 235 - 244.

[7] S. Haykin, Neural Networks, IEEE Press, New York, 1994.

[8] M. T. Hagan and M. B. Menhaj, "Training Feedforward Networks with the Marquardt Algorithm", IEEE Trans. on Neural Networks, Vol. 5, No. 6, 1994, pp. 989-993.

[9] FraukeGünther, Stefan Fritsch, "FraukeGünther and Stefan Fritsch", Research Article, The R Journal Vol. 2(1), June 2010, pp 3038.

[10] Thomas Behan, "Investigation of Integer Neural Networks for low cost embedded System”, in Electrical and Computer Engineering Commons", Jan 2009, pp 5-15.

[11] Thomas Behan, Zaiya Liao, Lian Zhao, Chunting Yang, "Accelerating Integer Neural Networks on Low Cost DSPs", World Academy of Science, Engineering and Technology 2008, pp 439-442.

[12] M. Sayaye-Pasand, H. Khorashadi-Zadeh, "Transmission Line Fault Detection and Phase Selection using ANN", in International Conference on Power System Transients, 2003, pp. 1-6.

[13] TaharBouthiba, "Artificial Neural Network-based Fault Location in EHV Transmission Lines", Electric Power System Research

[14] Aizenberg, N. Aizenberg and J. Vandewalle "Multi-valued and universal binary neurons: theory, learning, application"”, Kluwer Academic Publishers, Boston/Dordrecht/London, 2000.

[15] K. Hornik, M. Stinchcombe, H. White. "Multilayer Feedforward Neural Networks are Universal Approximators", Neural Networks, vol. 2, 1989, pp. 259-366.

[16] Seema Singh and T. V. Rama Murthy, "Neural Network based Sensor Fault Detection for Flight Control Systems", International Journal of Computer Applications (IJCA), Vol 59, No 13, Dec, 2012, pp 1-8.

[17] Seema Singh and T. V. Rama Murthy, "Neural Network based Sensor Fault Accommodation in Flight Control System, Journal of Intelligent systems (JISYS), De Gruyter, Vol 22, Issue 3, September, 2013, pp 317-333 